\title{
Advertising and competitive access pricing to internet services or pay-TV
}

J.J. Gabszewicz, D. Laussel and N. Sonnac

Discussion Paper 2006-44

\section{Département des Sciences Économiques de l'Université catholique de Louvain}




\title{
CORE Discussion Paper 2006/86
}

\section{Advertising and competitive access pricing to internet services or pay-TV}

\author{
Jean-Jaskold-Gabszewicz Didier Laussel \\ Nathalie Sonnac
}

September 29, 2006

\begin{abstract}
We study access pricing by platforms providing internet services ot pay-TV to users while they allow advertisers to have access to these users against payment via ads or banners. Users are assumed to be ad-haters. It is shown that equilibrium access prices in the users'market are increasing in the dimension of the advertising market: the larger the number of advertisers, the higher the access prices for both platforms.
\end{abstract}




\section{Introduction}

Consider a two-sided market where the platforms sell their product to a population of users (or consumers), while they sell to a population of advertisers, the opportunity of accessing to the users via ads or banners. This market context arises for instance when internet service providers give access to this service to a population of users against payment, or when pay -TV channels compete to attract viewers. Then a natural question to formulate is whether the access prices opposed by the platforms to users are negatively, or positively, correlated to the amount of advertising revenues: do users benefit via lower prices from the existence of an advertising market? This issue has been theoretically investigated in the monopoly case by Gabszewicz, Laussel and Sonnac (2005). They show that advertisers subsidize the users by lowering the access price whenever (i) there is a majority of users who are ad-lovers and ad-attraction is sufficiently large and (ii) there is a majority of ad-averse users and ad-repulsion is not too strong. Here we investigate the oligopoly case when all users are ad-averse, a case which seems to correspond to their natural behavior in the two situations evoked above, namely internet services and pay-TV.

In order to study this issue we develop a double vertical differentiation model where users differ in their degree of ad-aversion while advertisers differ in the benefits which they derive from advertising. We suppose that the services sold by the platforms are perfect substitutes from the users' viewpoint as far as the amounts of advertising are identical. Platforms are assumed to compete in prices in both markets. It is shown that the equilibrium access prices in the users'market (Internet access or pay-TV) are increasing in the dimension of the advertising market: the larger the number of advertisers, the higher the access prices for both platforms. Far from subsidizing the users, advertising thus generates an anti-competitive effect which is detrimental to consumers. This resembles the effect on competition of a negative consumption externality in the case of a simple network effect (see Grilo, Shy and Thisse (2001) or de Montmarin, Laussel and Long (2004)), but working here through intermarket negative externalities. As a by-product, we show that price equilibria are equal for the two competing platforms, a surprising feature in a model of vertical differentiation! Furthermore, we consider a variation of the model in which platforms compete in prices in the users' market and in quantities in the advertising market. Peitz and Valetti (2006) have considered a similar situation in a related model which differs from ours by the fact that firms are horizontally differentiated and users, while being ad-averse, do not differ from each other in their degree of ad-repulsion. These authors show that there is a full pass-through of advertising revenues into lower access prices. In our variant of the model, our result is that there exists a pure strategy Nash equilibrium iff the marginal cost in the users' market is zero. Indeed a Bertrand-like competition both in prices and qualities (i.e. the volumes of advertising) necessarily drives the profits to zero but, simultaneously, there always exists an advantageous deviation from the zero profit situation, either with higher price and higher quality, or with lower price and lower quality. 


\section{The Model}

We consider a two-sided market with a continuum of advertisers'types, with density $\alpha$, indexed by $\theta \in[0,1]$, a continuum of users indexed by $\mu \in[0,1]$ and two competing platforms (say Internet Service Providers or ISP's for short) offering identical services. Given this assumption, users always single-home: they buy access to one and only one of the two ISP's. We assume that the intrinsic value of having access to one ISP takes a sufficientlylarge value $U$ so that the net utility of any consumer is positive at equilibrium and the user's market is always covered. The net surplus derived by consumer $\mu$ from accessing to firm $i$ is assumed to be given by

$$
U-\mu a_{i}-p_{i}
$$

where $a_{i}$ is the number of advertisers who advertise at ISP $i(i=1,2)$. As is obvious from this formulation we suppose that users dislike advertising but differ in their degree of ad-aversion. Assuming that the number of users is normalized to 1 , for given numbers of advertisers $a_{1}$ and $a_{2}$ and given firms prices $p_{1}$ and $p_{2}$ the number of users of each firm is given by

$$
\begin{aligned}
& r_{1}=1-\frac{p_{1}-p_{2}}{a_{2}-a_{1}} \\
& r_{2}=\frac{p_{1}-p_{2}}{a_{2}-a_{1}}
\end{aligned}
$$

when $a_{2}>a_{1}{ }^{1}$ and by

$$
\begin{aligned}
& r_{2}=1-\frac{p_{2}-p_{1}}{a_{1}-a_{2}} \\
& r_{1}=\frac{p_{2}-p_{1}}{a_{1}-a_{2}}
\end{aligned}
$$

when $a_{2}<a_{1}{ }^{2}$. Of course, when when $a_{2}=a_{1}$ the two firms are perfect substitutes from the users' viewpoint with the obvious consequence that they always buy the less expensive of the two ${ }^{3}$.

The net utility which a type $\theta$-advertiser derives from inserting a banner at price $v_{i}$ at ISP $i$ is given by

$$
\theta r_{i}-v_{i}
$$

\footnotetext{
${ }^{1}$ Of course this holds true iff $\frac{p_{1}-p_{2}}{a_{2}-a_{1}} \in[0,1] \cdot \frac{p_{1}-p_{2}}{a_{2}-a_{1}}<0 \Rightarrow r_{1}=1, r_{2}=0$ while $\frac{p_{1}-p_{2}}{a_{2}-a_{1}}>$ $1 \Rightarrow r_{1}=0, r_{2}=1$.

${ }^{2}$ Of course this holds true iff $\frac{p_{2}-p_{1}}{a_{1}-a_{2}} \in[0,1] \cdot \frac{p_{2}-p_{1}}{a_{1}-a_{2}}<0 \Rightarrow r_{2}=1, r_{1}=0$ while $\frac{p_{2}-p_{1}}{a_{1}-a_{2}}>$ $1 \Rightarrow r_{1}=1, r_{2}=0$

${ }^{3}$ When, in addition, $p_{1}=p_{2}$, we suppose that $r_{1}=r_{2}=\frac{1}{2}$.
} 
Using an argument similar to the one used in Gabszewicz, Laussel and Sonnac (2002), and assuming that this utility is additive in case of multihoming, one can show that advertisers consider separately the opportunity of inserting banners at the two ISP's and that the demands for banners are given by

$$
a_{i}=\alpha\left(1-\frac{v_{i}}{r_{i}}\right), i=1,2
$$

Equivalently, the inverse demand functions for banners are given by

$$
v_{i}=\alpha r_{i} a_{i}\left(1-a_{i}\right) .
$$

Finally we assume that the marginal cost of providing access to the service to an extra user is equal to $c \geq 0$, whereas ad-costs for providers are supposed to be zero. In the case $c>0$, then the opportunity of subsidizing access in the users' market exists since it allows access prices which are strictly below marginal and average cost.

\section{Price Competition in the Advertising Market}

Suppose that users have rational expectations with respect to the number of banners, while advertisers hold rational expectations with respect to the number of users. Then we can solve for $r_{1}, r_{2}, a_{1}$ and $a_{2}$ as functions of prices $p_{1}, p_{2}$, $v_{1}$ and $v_{2}$. Assuming $a_{2}>a_{1}$, we obtain

$$
\begin{aligned}
r_{1} & =\frac{\alpha v_{1}}{p_{1}-p_{2}+\alpha\left(v_{1}+v_{2}\right)} \\
r_{2} & =\frac{p_{1}-p_{2}+\alpha v_{2}}{p_{1}-p_{2}+\alpha\left(v_{1}+v_{2}\right)} \\
a_{1} & =\alpha\left(1-v_{1}-v_{2}\right)-\left(p_{1}-p_{2}\right) \\
a_{2} & =\frac{\alpha\left[\alpha v_{2}\left(1-v_{1}-v_{2}\right)+\left(p_{1}-p_{2}\right)\left(1-v_{2}\right)\right]}{\alpha v_{2}+p_{1}-p_{2}} .
\end{aligned}
$$

When these solutions are non negative, it is easy to check that the condition $a_{2}>a_{1}$ is equivalent to $p_{1}>p_{2}{ }^{4}$. Assuming now $a_{2}<a_{1}$, we get

$$
\begin{aligned}
r_{1} & =\frac{p_{2}-p_{1}+\alpha v_{1}}{p_{2}-p_{1}+\alpha\left(v_{1}+v_{2}\right)} \\
r_{2} & =\frac{\alpha v_{2}}{p_{2}-p_{1}+\alpha\left(v_{1}+v_{2}\right)} \\
a_{1} & =\frac{\alpha\left[\alpha v_{1}\left(1-v_{1}-v_{2}\right)+\left(p_{2}-p_{1}\right)\left(1-v_{1}\right)\right]}{p_{2}-p_{1}+\alpha v_{1}} \\
a_{2} & =\alpha\left(1-v_{1}-v_{2}\right)-\left(p_{2}-p_{1}\right) .
\end{aligned}
$$

\footnotetext{
${ }^{4}$ We have indeed $a_{2}-a_{1}=\frac{\left(p_{1}-p_{2}\right)\left(p_{1}-p_{2}+\alpha\left(v_{1}+v_{2}\right)\right)}{p_{1}-p_{2}+\alpha v_{2}}$.
} 
When these solutions are non negative it is easy to check that the initial condition $a_{2}<a_{1}$ is equivalent to $p_{1}<p_{2}$. Thus we conclude that a larger price in the users' market entails a lower advertising demand, independently from the value of advertising prices. Both systems of equations (5) and (6) imply that demands tend toward the same limits when the difference in prices $\left(p_{1}-p_{2}\right)$ tends toward 0 , namely

$$
\begin{aligned}
r_{i} & =\frac{v_{i}}{v_{1}+v_{2}}, i=1,2 \\
a_{i} & =\alpha\left(1-v_{1}-v_{2}\right), i=1,2 .
\end{aligned}
$$

Hence, demands are continuous at $p_{1}=p_{2} \cdot{ }^{5}$ and, by continuity, they take the above values at this point.

We assume that the two platforms simultaneously and independently choose their strategy, namely a pair of prices, one in each market.

Proposition 1 When platforms compete in prices in both markets, there is a unique Nash equilibrium given by

$$
\left(p_{i}, v_{i}\right)=\left(c+\frac{3 \alpha}{16}, \frac{3}{8}\right), i=1,2 .
$$

Proof. see appendix

Notice that the equilibrium identified in Proposition 1 is symmetric in both prices: both are identical for the two competing platforms. Moreover, platforms' access prices are increasing in the (relative) size of the advertising market while the advertising prices remain constant. Intuitively, this conclusion follows from generalized ad-aversion. The existence of advertising dampens competition on the users' market, as shown by the following heuristic argument. Consider for instance a unilateral deviation by one platform from the common equilibrium price. A price decrease, say by platform 1 , does not move all users away from platform 2, despite the fact that the two platforms are perfect substitutes from the users' viewpoint. This is so because the expected increase in the number of users $r_{1}$ is rationally expected to induce an increase in the number of ads inserted in platform 1 which, in turn, makes it less attractive for its users. This effect is all the more strong as the size of the advertising market is larger. A simple corollary of Proposition 1 is that both platforms' profits are increasing in the size $\alpha$ of the advertising market. This follows both from a direct effect (the advertising demand increases with $\alpha$ ) and from a strategic effect (the users' prices increase with the size of the advertising market as explained above).

\footnotetext{
${ }^{5}$ Notice that they are not continuously differentiable at $p_{1}=p_{2}$.
} 


\section{A variant of the model: quantity competition in the advertising market}

When the platforms compete in prices in the readers and in quantities in the advertising market, i.e. they simultaneously and non cooperatively choose $\left(p_{1}, a_{1}\right)$ and $\left(p_{2}, a_{2}\right)$, the profit of platform $i$ is given by

$$
\pi_{i}=\left[p_{i}+\alpha a_{i}\left(1-a_{i}\right)-c\right] r_{i}
$$

One can show that

Proposition 2 There exists no Nash Equilibrium at which one or both firms make strictly positive profits

Proof. see appendix

The above proposition shows that when platforms are allowed to use the amount of advertising, instead of its price, as a component of their strategic choice, competition starts to become much harsher, and quite similar to Bertrand competition with homogeneous products. A firm has always the possibility of attracting all users by setting the same amount of advertising and a slightly lower price than its competitor.(or, alternatively, the same price and a slightly lower amount of advertising). In other words demand in the users 'market is discontinuous with respect to the access price and quantity of advertising variables at the point where prices and advertising levels are identical. However, since now each platform has a strategy with two strategic components, profitable deviations do still exist in a situation where both platforms earn zero profits. The deviating platform can either reduce its advertising supply and increase its price, or increase its advertising supply and reduce its price while retaining its customers (or at least some of them). Proposition 3 below shows that it is indeed the case whenever the marginal cost $c>0$. A pure strategy Nash equilibrium exists iff the marginal cost is zero. At this equlibrium the platforms do not advertise and set a zero access price.

Proposition $3 A$ Nash equilibrium in pure strategies exists iff $c=0$. It is then such that that $p_{i}=a_{i}=0, i=1,2$.

Proof. see appendix

It should be noticed that without a non-negativity constraint on prices (i.e. if users can be subsidized in one way or another) the non- existence of a pure strategy Nash equilibrium extends to the zero marginal cost case as well.

\section{References}

[1] Grilo, I., Shy, O. , Thisse, J., 2001, Price ompetition when onsumers behavior is characterized by conformity or vanity, Journal of Public Economics 80, 385-408. 
[2] de Montmarin, M., Laussel, D, and N.V. Long, 2004, Dynamic duopoly with congestion effects; International Journal of Industrial Organization, $22,655-677$

[3] Gabszewicz, J.J, Laussel, D, and N. Sonnac, 2005, Does advertising lower the price of newspapers to consumers? A theoretical appraisal; Economics Letters, 87, 2005, 127-134

[4] Peitz M. and T. Valetti, 2005, Content and advertising in the media: pay tv versus free-to-air; mimeo.

\subsection{Appendix}

\section{Proof of proposition 1 :}

(i) First, suppose that the equilibrium prices are such that $p_{1} \geq p_{2}$ and use equations (5). Choose $p_{1}$ and $v_{1}$ so as to maximize $\pi_{1}=p_{1} r_{1}+v_{1} a_{1}$ and, similarly, select $p_{2}$ and $v_{2}$ so as to maximize $\pi_{2}=p_{2} r_{2}+v_{2} a_{2}$. We obtain four equilibrium first-order conditions. This system can easily be solved using the change of variables $\rho_{i}=p_{i} / \alpha, i=1,2$, and substituting $\alpha \rho_{i}$ for $p_{i}$ in the equilibrium conditions. We then obtain four conditions in the four unknowns $\rho_{1}, \rho_{2}, v_{1}$ and $v_{2}$, conditions which do not depend anymore on $\alpha$. This system has a unique solution which satisfies our initial assumption. $p_{1} \geq p_{2}$ and which is given by $\rho_{1}=\rho_{2}=\frac{3}{16}$ and $v_{1}=v_{2}=\frac{3}{8}$ (corresponding of course to $\left.p_{1}=p_{2}=\frac{3 \alpha}{16}\right)$. For this solution to be an equilibrium, we have to show that the strategy $\left(\frac{3 \alpha}{16}, \frac{3}{8}\right)$ does indeed maximize platform i's profit when platform $\mathrm{j}$ has selected the pair of prices $\left(\frac{3 \alpha}{16}, \frac{3}{8}\right)$ when $p_{i}$ is constrained to exceed the value $p_{j}=\frac{3 \alpha}{16}$. For platform $i$, there exist two solutions derived from the first-order conditions in the admissible set of prices, namely, $\left(\frac{13 \alpha}{16}+4 \sqrt{13}, 0\right)$ and $\left(\frac{3 \alpha}{16}, \frac{3}{8}\right)$; furthermore, it is easy to check from the second-order conditions that only the second solution corresponds to a maximum. For platform $j$, the only solution in the set of admissible prices is $\left(\frac{3 \alpha}{16}, \frac{3}{8}\right)$ and, from the second-order conditions, it corresponds to a maximum of platform $j$ 's profit. (ii) Now suppose that the equilibrium prices are such that $p_{1} \leq p_{2}$ and consider equations (6). We may use the same argument as above in order to show that there exists a unique Nash equilibrium in this set of prices and that this equilibrium is also given by (7). Since this equilibrium is the same as above, it follows that it is the unique Nash equilibrium of the game. QED.

\section{Proof of proposition 2}

Suppose on the contrary that there exists a pure strategy Nash equilibrium $\left(\left(p_{i}^{*}, a_{i}^{*}\right),\left(p_{j}^{*}, a_{j}^{*}\right)\right)$ such that $\pi_{i} \geq \pi_{j}$ with $\pi_{i}>0$. There always exists in this 
case an arbitrarily small $\varepsilon>0$ such that firm $j$ by choosing $a_{j}=a_{i}^{*}$ and $p_{j}=$ $p_{i}^{*}-\varepsilon$ would attract all users (i.e. $\left.r_{j}=1\right)$ and earn a profit $\left[p_{i}^{*}-\varepsilon+\alpha a_{i}^{*}\left(1-a_{i}^{*}\right)\right]$ $>\pi_{i} \geq \pi_{j}$, a contradiction. QED.

\section{Proof of proposition 3}

(i) Let us first show that $((0,0),(0,0))$ is the unique pure strategy Nash equilibrium when $c=0$. Obviously there is no profitable deviation from this equilibrium since any feasible deviation would either imply a strictly positive price and/or a strictly positive advertising supply so that the deviating platform would lose all its customers and earn zero profits. So this pair of strategies is a Nash equilibrium. Straightforwardly from proposition 2, this is the only one.

(ii) From proposition 2, we have only to prove that when platform $j$ has selected any feasible strategy $\left(p_{j}, a_{j}\right)$ such that its profit per user $p_{j}+\alpha a_{j}(1-$ $\left.a_{j}\right)-c$ is equal to zero, there always exist a profitable deviation $\left(p_{i}, a_{i}\right)$ which gives platform $i$ a strictly positive profit. First consider a deviation by platform $i$ toward a higher quality (less advertising) and a higher price. For the sake of simplicity let us define $m_{k}=\frac{p_{k}-c}{\alpha}, k=i, j$. Using the zero profit assumption $m_{j}=-a_{j}\left(1-a_{j}\right)$, we can write the first-order conditions for platform $i$ as

$$
\begin{aligned}
\frac{-2 m_{i}+a_{i}^{2}+a_{j}^{2}-2 a_{i}}{a_{j}-a_{i}} & =0 \\
\frac{\left(1-2 a_{i}\right)\left(a_{i}-a_{j}\right)\left(a_{i}-a_{j}^{2}+m_{i}\right)+\left(-a_{i}+a_{i}^{2}-m_{i}\right)\left(a_{j}-a_{j}^{2}+m_{i}\right)}{\left(a_{j}-a_{i}\right)^{2}} & =0 .
\end{aligned}
$$

Solving with respect to $a_{i}$ and $m_{i}$ yields $a_{i}=\frac{a_{j}}{3}$ and $m_{i}=\frac{1}{9}\left(5 a_{j}^{2}-3 a_{j}\right)$, yielding a profit $\pi_{i}=\frac{8 a_{j}^{2}}{27}$ it follows that from any zero profit initial position there is a feasible profitable "high quality" deviation for platform $i$ whenever $a_{j}>0$. Let us now consider a "low quality " deviation toward more advertising space and lower prices. We write the first-order conditions as

$$
\begin{aligned}
\frac{a_{j}-a_{j}^{2}+a_{i}-a_{i}^{2}+2 m_{i}}{a_{j}-a_{i}} & =0 \\
\frac{\left(-a_{j}+a_{j}^{2}-m_{i}\right)\left(-a_{j}+2 a_{i} a_{j}-a_{i}^{2}-m_{i}\right)}{\left(a_{j}-a_{i}\right)^{2}} & =0
\end{aligned}
$$

Solving with respect to $a_{i}$ and $m_{i}$ yields $a_{i}=\frac{1+a_{j}}{3}$ and $m_{i}=\frac{1}{9}\left(5 a_{j}^{2}-5 a_{j}-1\right)$. yielding a profit $\frac{1}{27}\left(1-2 a_{j}\right)^{3}$. There is then a feasible profitable "low quality" deviation whenever $a_{j}<\frac{1}{2}$. We can conclude that there is always a feasible profitable deviation from a zero profit position, either toward less advertising and higher price or toward more advertising and lower price. QED. 
Département des Sciences Économiques de l'Université catholique de Louvain

Institut de Recherches Économiques et Sociales

Place Montesquieu, 3

1348 Louvain-la-Neuve, Belgique 